\title{
Dilated cardiomyopathy: diagnostic accuracy of endomyocardial biopsy
}

\author{
SUSUMU YONESAKA, * ANTON E BECKER \\ From the Department of Pathology, University of Amsterdam, Academic Medical Centre, Amsterdam, the \\ Netherlands
}

SUMMARY A histopathological index of contractility failure, which was reported to be accurate for the diagnosis of idiopathic dilated or congestive cardiomyopathy in Japan, was used to assess endomyocardial biopsy specimens from 41 Dutch patients with suspected dilated cardiomyopathy. The calculated index had a sensitivity of $62.5 \%$ and a specificity of $68 \%$ for idiopathic dilated or congestive cardiomyopathy. These disappointing results indicate that the histopathological index of contractility failure does not reliably distinguish between idiopathic dilated cardiomyopathy and other diseases that cause congestive heart failure.

It is a matter of debate whether or not endomyocardial biopsy specimens should be used for the diagnosis and management of patients with idiopathic dilated (congestive) cardiomopathy. ${ }^{1-3}$ The criteria set by the WHO/ISFC task force define the term "dilated cardiomyopathy" as a heart muscle disease of obscure origin with dilation of the ventricles. ${ }^{4}$ Decreased myocardial contractility is the prime mechanical disorder. ${ }^{5}$ The histopathological findings, such as myocyte hypertrophy, degeneration, interstitial fibrosis, and endocardial proliferative changes, are in themselves non-specific. ${ }^{6-9}$ The major contribution of an endomyocardial biopsy specimen, therefore, is to rule out other conditions that can cause congestive heart failure, such as idiopathic myocarditis. ${ }^{10}$ The existence of a relation between the features of endomyocardial biopsy specimens and prognosis is also controversial. Some workers found that prognosis was poor when myocardial fibrosis was widespread, ${ }^{711}$ but others did not find such a clear correlation

\footnotetext{
*Present address: Department of Paediatrics, Hirosaki University School of Medicine, 5 Zaifu-cho, Hirosaki-shi, Aomori-Ken, 036 Japan.
}

Requests for reprints to Professor Anton E Becker, Department of Pathology, Academic Medical Centre, Meibergdreef 9, 1105 AZ Amsterdam-Zuidoost, the Netherlands.

Accepted for publication 26 February 1987 between light microscopical and ultrastructural findings and the course of the disease. ${ }^{81213}$ An important reason for this discrepancy may be variation in the time of biopsy in relation to the stage of the disease. Biopsy specimens are usually taken after the onset of signs and symptoms and, hence, in most instances late in the course of the myocardial disease process. Indeed, inflammatory cell reactions were more common in those patients in whom the biopsy specimen was obtained early in the disease process. ${ }^{1415}$ This led to the assumption that idiopathic dilated cardiomyopathy may represent the end stage of a previous myocarditis. ${ }^{16}$

In view of these almost universally accepted findings it is surprising that Sekiguchi et al and Hiroe have claimed to have established histopathological criteria that enable them accurately to diagnose dilated cardiomyopathy on endomyocardial biopsy specimens irrespective of the stage of the disease. ${ }^{17-19}$ Sekiguchi et al and Hiroe have worked out a histopathological index of the failure of contractility, which they claim is a highly specific and sensitive method for the diagnosis of idiopathic dilated (congestive) cardiomyopathy.

Because this approach does not appear to have been evaluated outside Japan we used the Japanese criteria to study a series of endomyocardial biopsy specimens taken from patients in whom the initial clinical diagnosis was idiopathic dilated cardiomyopathy. 


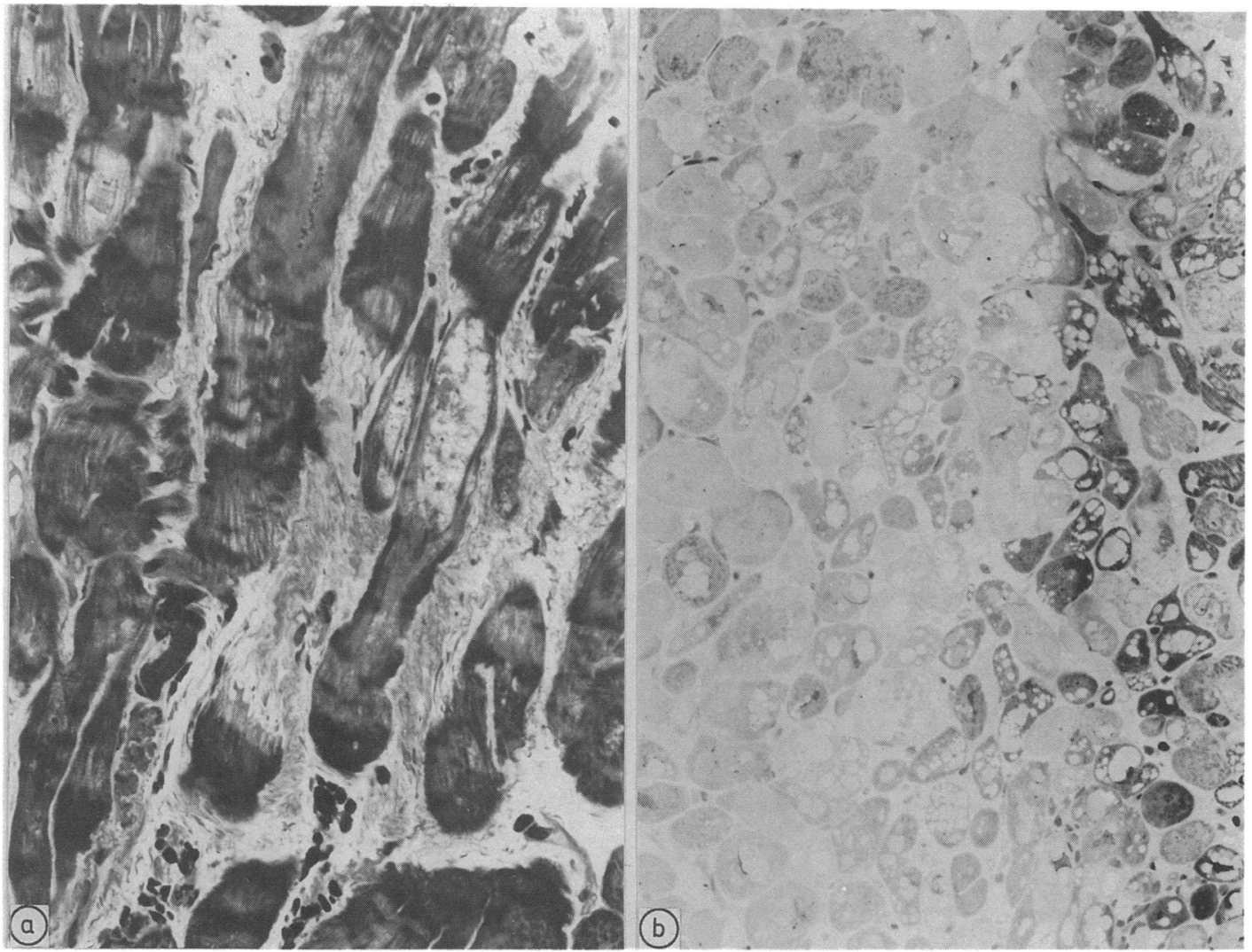

Fig 1 Photomicrographs of endocardial biopsy specimens from patients with idiopathic dilated cardiomyopathy showing degeneration and lysis of myocytes as defined by Sekiguchi et al ${ }^{1718}$ and Hiroe. ${ }^{19}$ Note the abundance of contraction bands, which are an artefact related to the procedure. (a) Mild (+) cytoplasmic vacuolisation with mild interstitial fibrosis.

(b) Severe lysis $(+++)$. The differences in staining intensity are related to embedding of the tissue in plastic.

Ultrastructural studies showed ischaemic changes with otherwise well preserved cellular organelles. Toluidine blue stain. (original magnification $a, \times 140 ; b, \times 64$ ).

\section{Patients and methods}

\section{ENDOMYOCARDIAL BIOPSY SPECIMENS}

We studied 41 patients ( 34 men and 7 women; aged 18-76, mean 42 years) in whom the initial clinical diagnosis was idiopathic dilated (congestive) cardiomyopathy. We studied a mean of five biopsy specimens from each patient; all were taken from the left ventricle. The specimens were fixed immediately in $2.5 \%$ phosphate buffered ( $\mathrm{pH} \mathrm{7.35)}$ glutaraldehyde and embedded in epon. Sections $0.5 \mu \mathrm{m}$ thick were cut and stained with an alkaline toluidine blue solution.

\section{HISTOLOGICAL ASSESSMENT}

The histological index of the failure of contractility was assessed according to Sekiguchi et $a l^{1718}$ and Hiroe ${ }^{19} \mathrm{We}$ followed the criteria set by the Japanese workers as accurately as possible; but this does not imply that we agree with their interpretation of the various histological and cytological features.

The index is based on three pathological features: degeneration and lysis of myocytes, interstitial fibrosis, and fragmentation of muscle bundles. Each of these histological features was scored from 0 to 3 (figs 1-3). The index was calculated by adding the score for each of the three histological features.

In addition, attention was given to attenuation of myocytes, smooth muscle cell hyperplasia in the endocardium, and changes in small vessels. The histological analysis was performed without knowledge of the clinical diagnosis.

\section{CIINICAI DIAGNOSIS}

The table shows the definitive diagnoses in 41 patients with an initial clinical diagnosis of idio- 


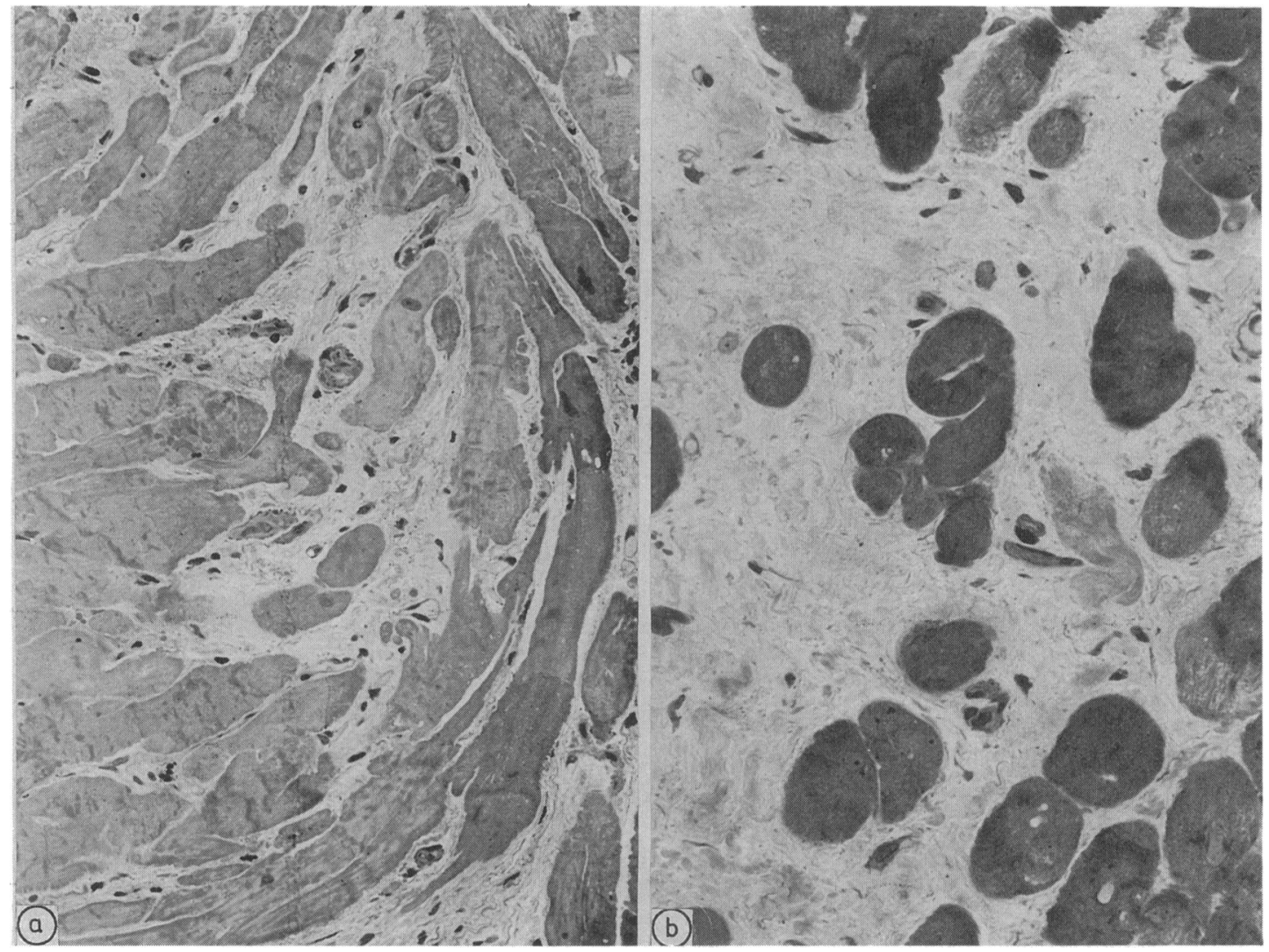

Fig 2 Photomicrographs showing interstitial fibrosis in endocardial biopsy specimens from patients with idiopathic dilated cardiomyopathy. (a) Mild fibrosis (+). The myofibres are mainly oriented longitudinally and contain contraction bands. (b) Severe fibrosis $(+++)$. The increased amount of fibrous tissue can be recognised despite the different orientation of the myocytes. Toluidine blue stain (original magnification $a$ and $b, \times 100$ ).

pathic cardiomyopathy. The clinical diagnosis of idiopathic dilated cardiomyopathy (16 patients) was based on collective data, including the patient's history, physical and laboratory examinations, chest $x$ rays, electrocardiographic tracings, echocardiography, radionuclide imaging, and cardiac catheterisation, including selective coronary arteriography. In each instance the clinical signs had been present for over six months and the patients all

Table Definitive clinical diagnoses

Idiopathic dilated cardiomyopathy

Idiopathic hypertrophic cardiomyopathy $(\mathrm{HCM})$

Arrhythmias:

Paroxysmal supraventricular tachycardia

Paroxysmal ventricular tachycardia

Hypertensive heart disease

Ischaemic heart disease

Valvar heart disease

Miscellaneous out of heart failure and fit for biopsy. The diagnosis idiopathic hypertrophic cardiomyopathy was based primarily on the clinical features defined by the WHO/ISFC task force. ${ }^{4}$ In the present series two patients had aortic and mitral valve insufficiency of uncertain origin. The six patients in the miscellaneous group had various conditions, such as rheumatoid heart disease, lymphoblastic leukaemia, acquired immune deficiency syndrome, and no cardiac abnormalities.

\section{Results}

Hypertrophy of myocytes was seen in all 41 patients but small vessel disease and inflammatory cell infiltration were not seen in any. Attenuation of the myocytes was seen in only two of the 16 patients with idiopathic dilated cardiomyopathy. Endocardial smooth muscle cell hyperplasia was seen in six of these 16 patients and in one each of the 


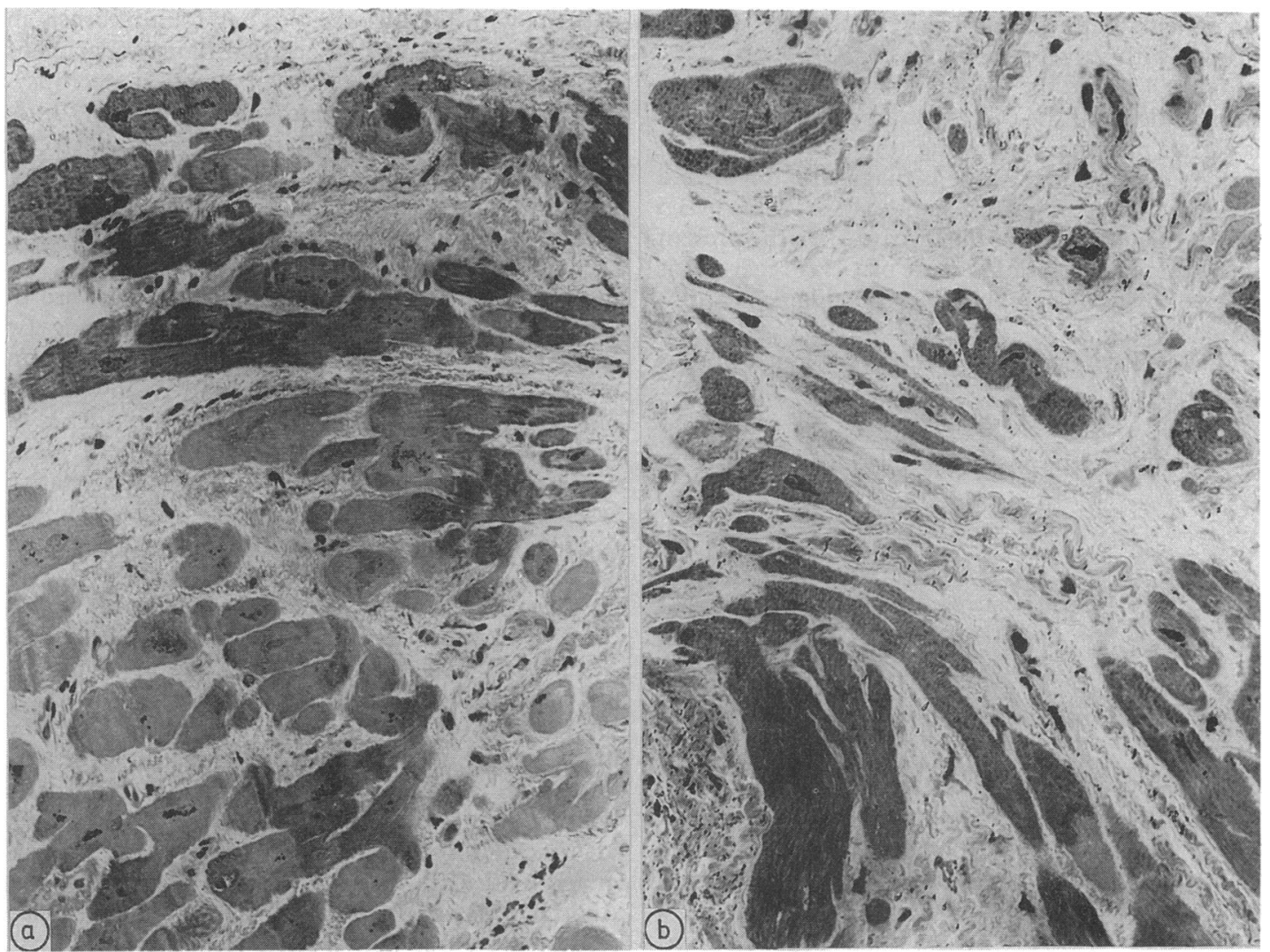

Fig 3 Photomicrographs showing fragmentation of muscle bundles in endocardial biopsy specimens from patients with idiopathic dilated cardiomyopathy. (a) Mild ( + ) and (b) severe $(+++)$. The term fragmentation includes fibres that taper out or "fragment" amidst fibrous tissue. Toluidine blue stain (original magnification a and b, $\times 100$ ).

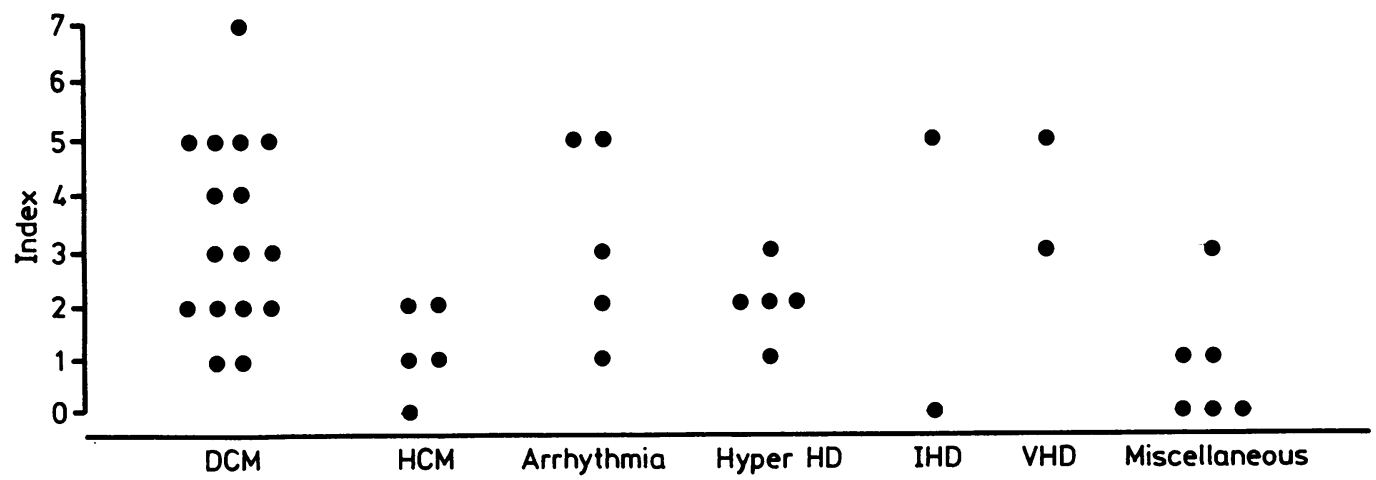

Fig 4 Histopathological index of failure of contractility calculated from endomyocardial biopsy specimens and corresponding clinical diagnoses. DCM, idiopathic dilated cardiomyopathy; HCM, hypertrophic cardiomyopathy; Hyper $H D$, hypertensive heart disease; IHD, ischaemic heart disease, VHD, valvar heart disease. 
five patients with primary arrhythmias and the two patients with valvar heart disease.

Figure 4 shows the histopathological contractility failure index and the corresponding clinical diagnosis for all patients. According to Sekiguchi and coworkers a high index score is diagnostic for dilated cardiomyopathy. They use a mean (SD) value of 3.5 $(1.4)$ as the indicator for the disease. ${ }^{19}$ In the present study, therefore, an index score of $\geqslant 3$ is regarded as positive histological evidence of dilated cardiomyopathy.

The sensitivity and specificity of the index were as follows:

$$
\begin{aligned}
\text { Sensitivity } & =\frac{\text { true positive }}{\text { true positive }+ \text { false negative }} \times 100 \\
& =\frac{10}{10+6} \times 100=62.5 \% \\
\text { Specificity } & =\frac{\text { true negative }}{\text { false positive }+ \text { true negative }} \times 100 \\
& =\frac{17}{8+17} \times 100=68 \%
\end{aligned}
$$

\section{Discussion}

The diagnosis idiopathic dilated or congestive cardiomyopathy is difficult because it is based mainly on the exclusion of known causes of heart failure rather than on positive criteria. It is likely that the disease has a multifactorial origin with a final common pathway characterised by decreased myocardial contractility. From this it is understandable that the histopathology in the later stages of the disease will be non-specific and, from the point of view of diagnosis, not very helpful. Baandrup et al did not find a significant correlation between the histological features and the clinical diagnosis in a series of 361 endomyocardial biopsy specimens from patients with suspected cardiomyopathy.$^{13}$ Moreover, they were unable to confirm the congestive nature of the underlying heart disease on the basis of the biopsy specimens alone and concluded that biopsy specimens did not indicate the prognosis. Moreover, in a retrospective study, they found no relation between ultrastructural findings and prognosis. ${ }^{20}$ When Breithardt and co-workers used a scoring system that resembled the one used by Baandrup et al they too concluded that the results of long term follow up could not be accurately established from biopsy specimens. ${ }^{12}$ On the other hand, myocyte degeneration appeared to be a prognostically important factor in patients with a history of less than two years. More recently, Rose and Beck examined 76 endomyocardial biopsy specimens from patients with the clinical diagnosis of dilated cardiomyopathy and 54 necropsy specimens from patients with this disease. ${ }^{21}$ They found fibrosis in only $28 \%$ of the endomyocardial biopsy specimens and in $50 \%$ of the sections obtained at necropsy. Moreover, they found remarkably little myocytolysis-none in the endomyocardial biopsy specimens and only $9 \%$ in the necropsy series. These workers also concluded that the clinical evaluation of cardiac dysfunction correlated poorly with the morphology of the myocardial tissue. The findings of Rose and Beck, ${ }^{21}$ in particular, are in striking contrast with the observations made by Sekiguchi et al, who showed $100 \%$ fibrosis both in the endomyocardial biopsy specimens and in the necropsy specimens and a much higher frequency of degeneration, including myocytolysis. In fact, Sekiguchi et al emphasised that a semiquantitative assessment of the endomyocardial biopsy specimens predicted the outcome in patients with dilated cardiomyopathy. ${ }^{17-19}$

Why do workers in different parts of the world come to such divergent conclusions? We followed Sekiguchi's method as closely as possible. This was not easy because the precise criteria on how to apply the grading system from $\mathbf{0}$ to $\mathbf{3}$ for each of the key items - that is degeneration and lysis of myocytes, interstitial fibrosis, and fragmentation of muscle bundles-are not clearly stated. Moreover, identification of the histopathological features promoted by the Japanese workers is highly subjective. Another important criticism is that the changes may represent various non-specific responses to injury, such as ischaemia. For instance, the degenerative changes that they assess are for the most part myofibrillar degeneration as described by Reichenbach and Benditt ${ }^{22}$ (fig 1a). Also vacuolar degeneration (fig $1 \mathrm{~b}$ ) is highly suggestive of subendocardial ischaemia as described by Geer et al. ${ }^{23}$ The changes regarded as typical of fragmentation of myocytes may be partly the result of orientation of myocytes out of the plane of section and fibrosis. In the present study, however, these changes were categorised as "fragmentation". Despite our reservations about the histological interpretation of these features we followed the Japanese criteria as closely as possible to ensure that the two studies were comparable.

We found that histological assessment did not allow a clear and unambiguous differentiation of the various clinical subgroups or of individual patients. Nevertheless, the histopathological index was much higher in patients with dilated cardiomyopathy (3.3) than in those with hypertrophic cardiomyopathy (1.2). In patients with arrhythmia, valvar heart disease, ischaemic cardiomyopathy, and heart disease secondary to long lasting hypertension, however, the histopathological contractility failure index was in 
the range of that found in dilated cardiomyopathy. Moreover, when an index score of three was taken to indicate the diagnosis of dilated cardiomyopathy the sensitivity was $62.5 \%$ and the specificity was $68 \%$.

We found that the histopathological contractility failure index was not a reliable tool in diagnosing dilated cardiomyopathy. The results suggest that the index reflects cardiac muscle damage that is not necessarily specific to idiopathic dilated cardiomyopathy. This conclusion accords with the nonspecific histological criteria of the index and the current hypothesis that dilated cardiomyopathy is not an entity in the strict sense but rather a multifactorial disease with a final common pathway leading to heart failure.

We thank Dr G K David and Dr D R Düren (Academic Medical Centre, Amsterdam), Dr J J R M Bonnier (Catharina Ziekenhuis, Eindhoven), and Dr J J M L Chappin (St Elisabeth Ziekenhuis, Tilburg) for supplying us with the biopsy specimens and for informing us of final clinical diagnoses.

SY was supported by a grant from the Hirosaki University School of Medicine, Hirosaki, Japan.

\section{References}

1 Johnson RA, Palacios I. Dilated cardiomyopathies of the adult (first of two parts). $N$ Engl $J$ Med 1982;307:1051-8.

2 Johnson RA, Palacios I. Dilated cardiomyopathies of the adult (second of two parts). N Engl J Med 1982;307:1119-26.

3 Fowles RE, Mason JW. Role of cardiac biopsy.in the diagnosis and management of cardiac disease. Prog Cardiovasc Dis 1984;27:153-72.

4 Report of the WHO/ISFC task force on the definition and classification of cardiomyopathies. $\mathrm{Br}$ Heart $\mathrm{J}$ 1980;44:672-3.

5 Hirota Y, Shimizu G, Kaku K, Saito T, Kawamura K. Mechanisms of compensation and decompensation in dilated cardiomyopathy. Am J Cardiol 1984;54: 1033-8.

6 Olsen EGJ. The pathology of cardiomyopathies. A critical analysis. Am Heart J 1979;98:385-92.

7 Shirey EK, Proudfit WL, Hawk WA. Primary myocardial disease. Correlation with clinical findings, angiographic and biopsy diagnosis. Am Heart J 1980;99:198-207.

8 Unverferth DV, Fetters JK, Unverferth BJ, et al. Human myocardial characteristics in congestive heart failure. Circulation 1983;68:1194-200.

9 Davies MJ. The cardiomyopathies: a review of terminology, pathology and pathogenesis. Histopathology 1984;8:363-93.

10 Mason JW, Billingham ME, Ricci DR. Treatment of acute inflammatory myocarditis assisted by endomyocardial biopsy. Am J Cardiol 1980;45:1037-44.

11 Kunkel B, Lapp H, Kober G, Kalgenbach M. Cor- relations between clinical and morphologic findings and natural history in congestive cardiomyopathy. In: Kaltenbach M, Loogen F, Olsen EGJ, eds. Cardiomyopathy and myocardial biopsy. Berlin: Springer Verlag, 1978:271-83.

12 Breithardt G, Kuhn H, Knieriem HJ. Prognostic significance of endomyocardial biopsy in patients with congestive heart failure. In: Kaltenbach $\mathbf{M}$, Loogen F, Olsen EGJ, eds. Cardiomyopathy and myocardial biopsy. Berlin: Springer Verlag, 1978: 258-70.

13 Baandrup U, Florio RA, Rehahn M, Richardson PJ, Olsen EGJ. Critical analysis of endomyocardial biopsies from patients suspected of having cardiomyopathy. II. Comparison of histology and clinical haemodynamic information. $\mathrm{Br}$ Heart $J$ 1981;45: 487-93.

14 Fenoglio JJ, Ursell PCh, Kellogg CF, Drusin RE, Weiss MB. Diagnosis and classification of myocarditis by endomyocardial biopsy. $N$ Engl $J$ Med 1983;308:12-8.

15 Dec GW, Palacios IF, Fallon JT, et al. Active myocarditis in the spectrum of dilated cardiomyopathies. N Engl J Med 1985;312:885-90.

16 Subramanian $R$. The pathology of myocarditis and cardiomyopathy. In: Robinson JA, O'Connell JB, eds. Myocarditis: precursor of cardiomyopathy. Lexington, Massachusetts, Toronto: The Collamore Press, 1983:129-40.

17 Sekiguchi M, Hiroe M, Morimoto S, Kawagoe Y. The contribution of endomyocardial biopsy to the diagnosis and assessment of cardiomyopathies. In: Hayase S, Murao S, eds. International congress series no 470. Proceedings of the VIII World Congress of Cardiology. Amsterdam: Excerpta Medica, 1979:583.

18 Sekiguchi M, Hiroe M, Morimoto S. On the standardization of histopathological diagnosis and semiquantitative assessment of the endocardium obtained by endomyocardial biopsy. Bull Heart Inst Jpn 1979-1980:55-85.

19 Hiroe M. Evaluation of left ventricular function and prognosis in patients with idiopathic cardiomyopathy in comparison with endomyocardial biopsy findings with semiquantitative histopathological analysiscomparative study of hypertrophic and congestive (dilated) cardiomyopathy. [In Japanese] $J$ Tokyo Wom Med Coll 1982;52:292-306.

20 Baandrup U, Florio RA, Roters F, Olsen EGJ. Electron microscopic investigation of endomyocardial biopsy samples in hypertrophy and cardiomyopathy. Circulation 1981;63:1289-98.

21 Rose AG, Beck W. Dilated (congestive) cardiomyopathy: a syndrome of severe cardiac dysfunction with remarkably few morphological features of myocardial damage. Histopathology 1985;9:367-79.

22 Reichenbach DD, Benditt EP. Myofibrillar degeneration. A response of the myocardial cell to injury. Arch Pathol 1968;85:189-99.

23 Geer JC, Crago CA, Little WC, Gardner LL, Bishop SP. Subendocardial ischemic myocardial lesions associated with severe coronary atherosclerosis. $\mathrm{Am} \mathrm{J}$ Patho! 1980;98:663-80. 